\title{
Comparison of the prognostic value of prognostic nutritional index in colorectal cancer deaths:a retrospective cohort study
}

\author{
Hongqi ZHOU ${ }^{1}$, Wenling WANG ${ }^{2 *}$ (D), Ruyi ZHANG ${ }^{3}$, Hongmin DONG ${ }^{2}$, Gang WANG², Weiwei CHEN², Guodong LI $^{2}$
}

\begin{abstract}
Evidences showed that Prognostic Nutritional Index (PNI) is one important predictive factor for some cancers. However, the association between PNI and colorectal cancer is still unclear. In this study, we aim to explore the association between PNI and death in patients with locally advanced colorectal cancer. We enrolled 400 patients with colorectal cancer. The pathological results was used to confirm it. The patients received radical resection. The PNI at baseline was set as the exposure variable, death was the outcome variable, and other potential confounders were also analyzed. There was no association between PNI and the deaths of local advanced colorectal cancer and radical resection of colorectal cancer (HR=0.88; 95\% CI, 0.78-1.00). Applying the cubic spline function, we found that it is not linear between PNI and death. Two-piecewise cox model showed that there was $68 \%$ decrease of risk of deaths when PNI change per $1(\mathrm{HR}=0.32 ; 95 \% \mathrm{CI}, 0.13-0.79)$ on the left side of the inflection point (41.35). On the right of the inflection point, there was no association between PNI and death. PNI and deaths are linear correlation. Within a certain range (PNI=26-41), PNI is an independent protective factor for death risk.
\end{abstract}

Keywords: prognostic nutritional index; colorectal cancer; prognosis.

Practical Application: We found that there is not linear relationship between prognostic nutritional index (PNI) and deaths. In a specific range, PNI can be used to predict the death risk of colorectal cancer.

\section{Introduction}

Colorectal cancer is one common cancer worldwide (Yiu \& Yiu, 2016).There was increasing trend with living standard improvement and lifestyle changes (Zhou et al., 2017). The prognosis of colorectal cancer is dependent on various factors. The Established prognostic factors include tumor grad, venous or lymphatic invasion (Zlobec \& Lugli, 2008; Allegra et al., 2009). These prognosis tools are very useful in clinical management.

Malnutrition is a significant public health problem worldwide. Studies showed that up to $50 \%$ of adults admitted to hospital suffered from malnutrition. Malnutrition is associated with decreased immune system function, impaired respiratory function and poor wound healing (Cheng et al., 2017). Malnutrition also affects prognosis in many groups of patients (Lukaski et al., 2017).

The prognostic nutritional index (PNI) is calculated from the serum albumin concentration and total lymphocyte count, which is a simple and objective indicator malnutrition (Cheng et al., 2017). The PNI was firstly employed to evaluate the nutritional status of surgical patients (Onodera et al., 1984). Subsequent studies suggest that PNI is also a potential prognostic factor for some cancers (Sun et al., 2014; Chan et al., 2015; Kanda et al., 2011; Fu et al., 2016; Jeon et al., 2016; Migita et al., 2013). However, it is still not clear whether the PNI is related to the prognosis of colorectal cancer.

In the present study, we controlled other confounders and assess the possibility of PNI at baseline as prognostic value of colorectal cancer deaths.

\section{Materials and methods}

\subsection{Participants}

In this study, we non-selectively and consecutively collected 412 patients with stage II-III colorectal cancer from the authors' affiliation from January 2013 to April 2018. The study was approved by the Ethics Committee of the authors' affiliation. The diagonosis and staging criteria are based on the American Cancer Society's seventh edition of the colorectal cancer TNM staging system.

Inclusion criteria included radical resection of colorectal cancer ( $\mathrm{R} 0$ resection), postoperative adjuvant therapy an staged stage II-III colorectal cancer according to postoperative pathological diagnosis report. Exclusion criteria included preoperative neoadjuvant radiotherapy and chemotherapy, combination with other malignant tumors, severe complications and deaths during perioperative period. 


\subsection{The collection of clinical informations}

The clinical informations were reviewed, including surgery or radiotherapy and chemotherapy, chest and abdomen CT, colorectaloscopy from the hospital's electronic medical record system. The survival data were from patient medical follow-up records and telephone follow-up. The follow up was carried out every three months. No follow-up records and follow-up time less than 6 months were also excluded. Chest and abdomen CT, colorectaloscopy, tumor markers, blood routine, blood biochemistry and patient symptoms and signs were recorded in follow up.

\subsection{Analysis of PNI and other covariates}

We collected parameters for calculating PNI at baseline. Based on the results, the formula (Nozoe et al., 2012) was calculated as $\mathrm{PNI}=$ serum albumin value $(\mathrm{g} / \mathrm{L})+5 \times$ total number of peripheral blood lymphocytes $\left(\mathrm{x} 10^{9} / \mathrm{L}\right)$.

Other covariates related to death of PNI and colorectal cancer patients were also analyzed, including age, platelet count, tumor markers (CEA, CA199, CA724, CA242).Categorical variables: gender, survival status, tumor location, histological type, degree of differentiation, TNM staging, and postoperative treatment (Chang et al., 2013; Yang et al., 2016; Akgül et al., 2017; Zhao et al., 2018; Peng et al., 2017; Mohri et al., 2013).

\subsection{Statistical analyses}

Differences in the distribution of baseline characteristics between different PNI groups (TERTILE) were examined using $\chi^{2}$ (categorical variables), one-way ANOVA (normal distribution) or Kruskal-Wallis $\mathrm{H}$ (skew distribution).The categorical variables are expressed in terms of frequency or percentage, and the continuous variables are expressed as mean \pm standard deviation (normal distribution) or median (skewed distribution).We used univariate and multivariate Cox regression models to analyze the relationship between exposure factors and outcomes in colorectal cancer patients. Based on the recommendations of the STROBE statement (Vandenbroucke et al., 2014), we built three models: no covariates were adjusted, micro-adjusted models (adjusted age, gender only) and all covariates presented in Table 1. To address nonlinearity, The cubic spline function is applied to the model ( $\mathrm{R}$ rms package) for curve fitting. The cubic

Table 1. The correlation between PNI status and various of clinicopathological factors.

\begin{tabular}{|c|c|c|c|c|c|}
\hline & Low-PNI group & Middle-PNI group & High-PNI group & $\mathrm{P}^{*}$-value & $\mathrm{P}^{*}$-value \\
\hline $\mathrm{N}$ & 132 & 134 & 134 & & \\
\hline Age (years) & $56.94(12.60)$ & $55.22(11.89)$ & $51.53(13.38)$ & 0.002 & 0.005 \\
\hline PLT & $249.02(108.87)$ & $233.20(85.68)$ & $240.43(85.81)$ & 0.390 & 0.609 \\
\hline CA199 & $10.96(0.01-700.00)$ & $10.54(0.32-9028.00)$ & $11.72(0.00-700.00)$ & 0.572 & 0.611 \\
\hline CA72-4 & $2.10(0.47-52.76)$ & $2.19(0.53-300.00)$ & $2.17(0.44-300.00)$ & 0.536 & 0.902 \\
\hline CA242 & $4.94(0.58-310.51)$ & $4.86(0.01-129.73)$ & $5.15(0.10-300.00)$ & 0.095 & 0.860 \\
\hline Sex & & & & 0.918 & - \\
\hline Male & $82(62.12 \%)$ & $80(59.70 \%)$ & $81(60.45 \%)$ & & \\
\hline Female & $50(37.88 \%)$ & $54(40.30 \%)$ & $53(39.55 \%)$ & & \\
\hline Living state & & & & 0.037 & - \\
\hline Survive & $112(86.61 \%)$ & $128(95.38 \%)$ & $124(92.42 \%)$ & & \\
\hline Colon & $59(44.70 \%)$ & $50(37.31 \%)$ & $45(33.58 \%)$ & & \\
\hline Histological type & & & & 0.271 & 0.140 \\
\hline Adenocarcinoma & $119(90.15 \%)$ & $128(95.52 \%)$ & $120(90.23 \%)$ & & \\
\hline Mucinous adenocarcinoma & $13(9.09 \%)$ & $6(3.73 \%)$ & $14(9.77 \%)$ & & \\
\hline TNM stage & & & & 0.001 & - \\
\hline II & $53(40.15 \%)$ & $79(58.96 \%)$ & $52(38.81 \%)$ & & \\
\hline III & $79(59.85 \%)$ & $55(41.04 \%)$ & $82(61.19 \%)$ & & \\
\hline Postoperative treatment & & & & 0.295 & - \\
\hline Radical resection only & $12(8.73 \%)$ & $18(11.28 \%)$ & $11(7.69 \%)$ & & \\
\hline $\begin{array}{l}\text { Postoperative concurrent chemoradiotherapy } \\
\text { and adjuvant chemotherapy }\end{array}$ & $117(64.29 \%)$ & $120(70.68 \%)$ & $122(73.85 \%)$ & & \\
\hline
\end{tabular}

$\mathrm{P}^{*}$ indicated Nonparametric test. Based on the purpose of sensitivity analysis, we present the results of both parametric and nonparametric tests for continuous variables. The results are all robustness. 
spline function can study the nonlinear effects of continuous covariates and flexibly evaluate the interactions between covariates (Heinzl \& Kaider, 1997). In the case of non-linear correlation, we first calculated inflection using recursive algorithm, and then performed a two-part Cox regression model to establish regression equations at both ends of the inflection point to calculate the saturation-threshold effect of PNI on survival. We choose the best-fit model (two-piecewise cox model vs standard cox model) according to P for log likelihood ratio test (Liu et al., 2013). We also did the following to see if the result is robustness. We handle PNI as categorical variables (tertile, low, middle, high) and calculate $P$ for trend to see if the result is consistent with the PNI as a continuous variable. Statistical software package R (http://www.R-project.org, The R Foundation) and EmpowerStats (http://www.empowerstats.com, X\&Y Solutions, Inc, Boston, MA) were employed to analyze the results.

\section{Results}

\subsection{Patients' characteristics}

412 patients with stage II-III colorectal cancer were enrolled to this study. 2 patients received preoperative neoadjuvant chemoradiation and were excluded from the study. 10 patients were excluded due to no follow-up or less than 6 month follow up. The data from 400 patients were further analyzed (Figure 1).

\subsection{Association between PNI and survival}

The median follow-up time was 30 months, the shortest follow-up time was 6 months, and the longest follow-up time was 82 months. The median survival time was 32 months. Survival curves were constructed by Kaplan-Meier method. There was no significant difference in the cumulative overall survival rate between the PNI high-value group and the median group. However, both were significantly higher than the PNI low-value group $(\mathrm{P}=0.028)$ (Figure 2).

\subsection{Comparison of other covariates for different PNI level in colorectal cancer patients}

For the 400 patients, 154 cases received colon cancer radical resection and 246 cases received rectal cancer radical resection. Postoperative pathology showed that 184 cases were at stage II and 216 cases were at stage III. After converting exposure variables (PNI values for 400 patients) from continuous variables to categorical variables, the samples were divided into three groups according to PNI value: low value group, median group and high value group. There were 132 patients in the PNI low-value group, 134 patients in the PNI median group and 134 patients in the PNI high-value group. The preoperative PNI was $49.05 \pm 5.51$ (a minimum of 26.95 and a maximum of 68.2). Other covariates, such as age, platelet count, CA199, CA724, CA242, CEA, follow-up time, gender, survival status, tumor location, histological type, differentiation degree, TNM staging, postoperative treatment and differentiation degree Features were also grouped and compared for differences in PNI values (Table 1). The results showed that platelet count, tumor markers CA199,CA724,CA242,CEA,follow-up time, gender, tumor location, histological type, degree of differentiation and postoperative treatment were not different among the different PNI values. Age, tumor stage and patient survival were different among the different PNI groups. The age $(\mathrm{P}=0.002, \mathrm{P}=0.005)$ was decreased with the increase of the age. The survival status $(\mathrm{P}=0.037)$ and TNM stage II $(\mathrm{P}=0.001)$ was the highest in the median PNI group.

\subsection{Univariate analysis of the related factors associated with overall survival time in colorectal cancer}

The Cox regression univariate analysis model was used to analyzed the tumor prognosis marker. CA242 (HR, 1.01; 95\% CI 1.00-1.01; $\mathrm{P}=0.0030$ ), CEA (HR, 1.02; 95\% CI 1.01-1.03; $\mathrm{P}=0.0042)$, stage III (HR, 4.73; 95\% CI 1.83-12.27; $\mathrm{P}=0.0014$ ) were risk factors for death in colorectal cancer patients. Gender, age, platelet count, CA199, CA72-4, PNI value, tumor location, histological type, postoperative treatment, and degree of differentiation were not significantly associated with overall survival in patients with colorectal cancer (Table 2).

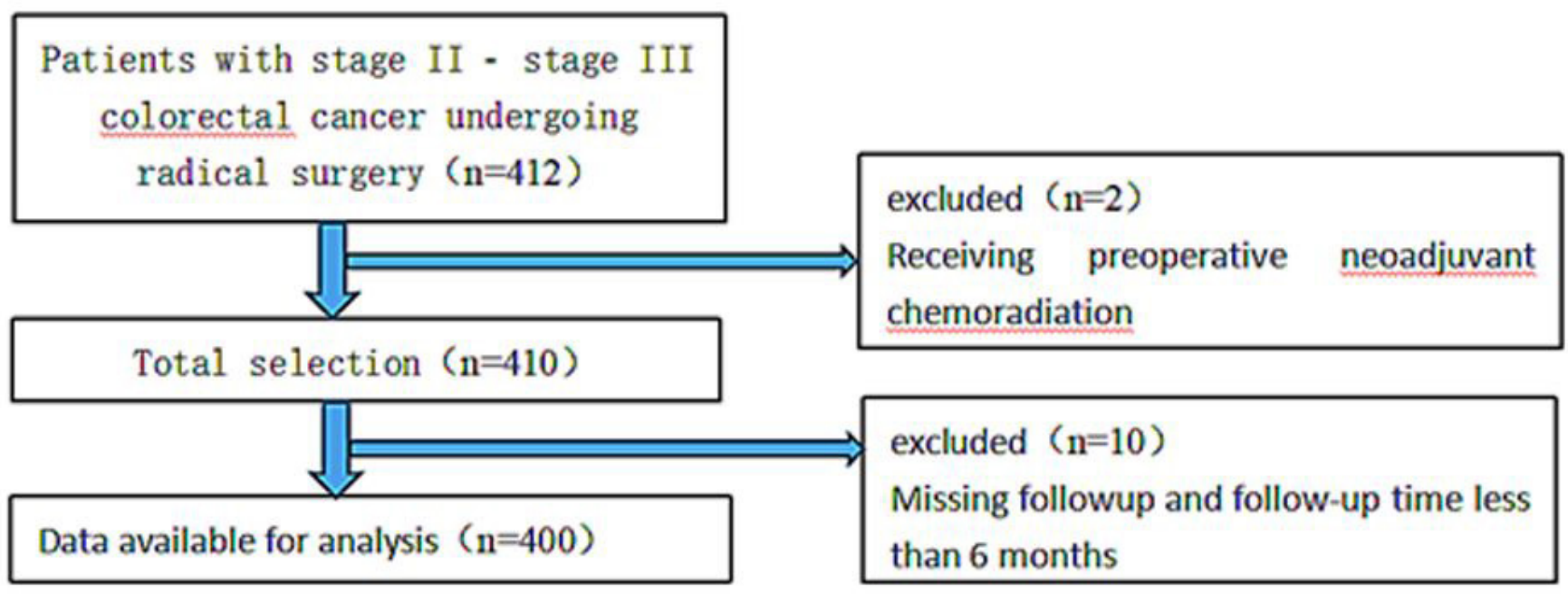

Figure 1. Study enrollment flow chart. 


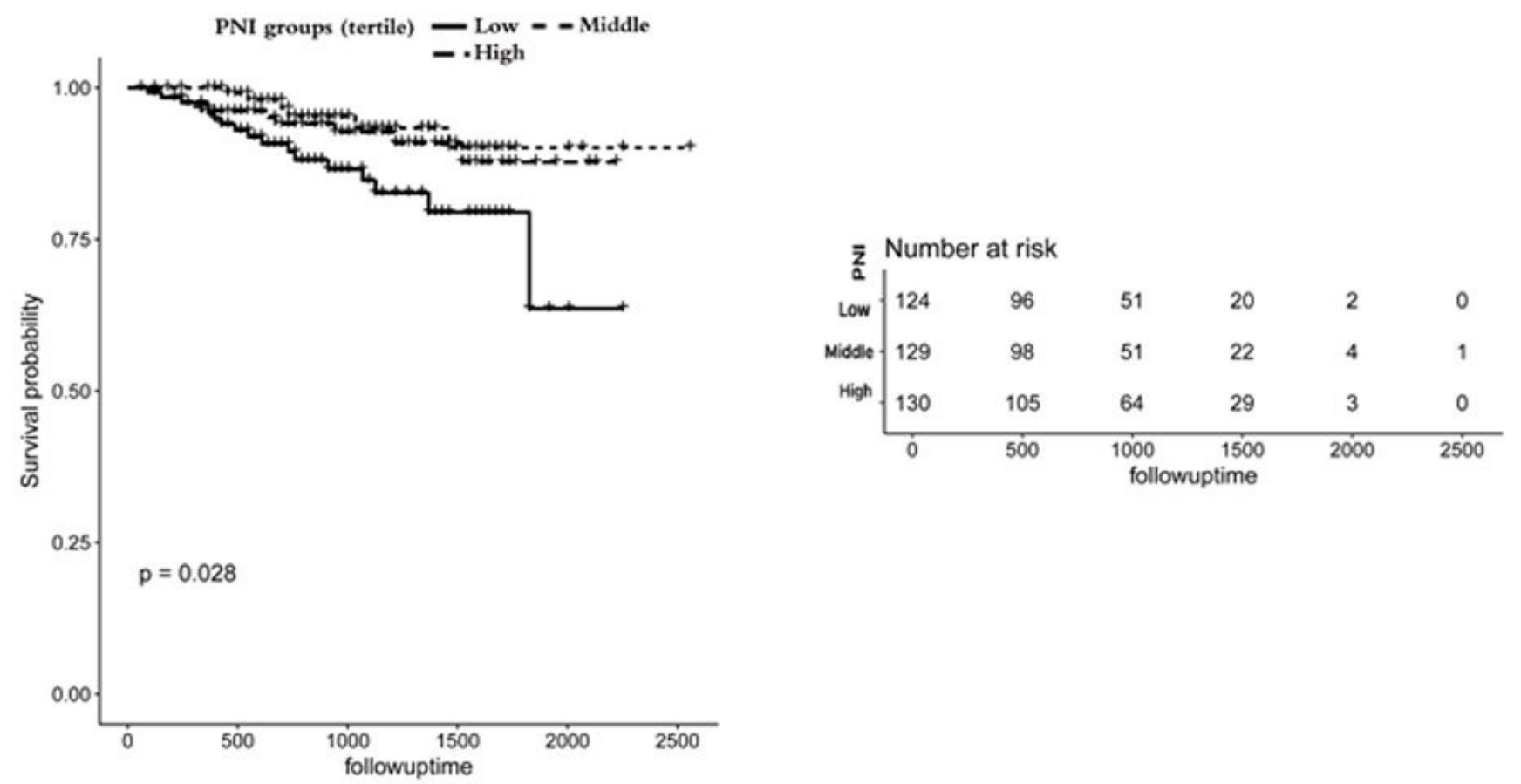

Figure 2. Total survival time curve of patients with colorectal cancer.

Table 2. Univariate analysis of the overall survival time of patients with colorectal cancer.

\begin{tabular}{|c|c|c|c|}
\hline & Ststistics & HR 95\% CI (lower, upper) & $\mathrm{p}$ values \\
\hline \multicolumn{4}{|l|}{ Sex } \\
\hline Male & $243(60.66 \%)$ & 1.0 & \\
\hline Female & $157(39.34 \%)$ & $1.31(0.66,2.60)$ & 0.4415 \\
\hline Age (years) & $54.58+12.86$ & $1.01(0.98,1.04)$ & 0.5424 \\
\hline PLT & $240.34+93.85$ & $1.00(1.00,1.00)$ & 0.9607 \\
\hline CA199 & $49.53+462.26$ & $1.00(1.00,1.00)$ & 0.9119 \\
\hline CA72-4 & $9.02+34.15$ & $1.01(1.00,1.01)$ & 0.0993 \\
\hline CA242 & $15.37+39.81$ & $1.01(1.00,1.01)$ & 0.0030 \\
\hline CEA & $6.19+14.62$ & $1.02(1.01,1.03)$ & 0.0042 \\
\hline PNI value & $49.05+5.51$ & $0.94(0.89,1.00)$ & 0.0607 \\
\hline \multicolumn{4}{|l|}{ Tumor location } \\
\hline Rectum & $246(62.03 \%)$ & 1.0 & \\
\hline Colon & $154(37.97 \%)$ & $0.67(0.31,1.45)$ & 0.3098 \\
\hline \multicolumn{4}{|l|}{ Histological type } \\
\hline Adenocarcinoma & $367(92.11 \%)$ & 1.0 & \\
\hline Mucinous adenocarcinoma & $33(7.38 \%)$ & $1.53(0.54,4.34)$ & 0.4290 \\
\hline \multicolumn{4}{|l|}{ TNM stage } \\
\hline II & $184(46.45 \%)$ & 1.0 & \\
\hline III & $216(53.55 \%)$ & $4.73(1.83,12.27)$ & 0.0014 \\
\hline \multicolumn{4}{|l|}{ Postoperative treatment } \\
\hline Radical resection only & $41(9.14 \%)$ & 1.0 & \\
\hline Postoperative concurrent chemoradiotherapy and adjuvant chemotherapy & $359(69.45 \%)$ & $1.54(0.32,7.44)$ & 0.5880 \\
\hline \multicolumn{4}{|l|}{ Differentiation } \\
\hline Well/moderate & $349(88.25 \%)$ & 1.0 & \\
\hline Poor & $51(11.75 \%)$ & $1.10(0.38,3.16)$ & 0.8633 \\
\hline
\end{tabular}

\subsection{The results of unadjusted and adjusted models}

Cox regression models were used to analyze whether different PNI values were associated with prognosis. The unadjusted and adjusted models were shown in Table 3. PNI values were found in the unadjusted model $(\mathrm{HR}=0.94,95 \%$ confidence interval $(\mathrm{CI})$ : 0.89-1.00, $\mathrm{p}=0.061)$. There was no significant change in HR trends $(\mathrm{HR}=0.95,95 \% \mathrm{CI}: 0.89-1.01, \mathrm{p}=0.078)$ in the minimallyadjusted model compared to the unadjusted model. In a fully 
adjusted model (HR=0.88, 95\%CI: 0.78-1.00, $\mathrm{p}=0.051)$, we adjusted histological type, gender, age, platelet count, TNM stage, postoperative treatment, degree of differentiation, tumor Markers and tumor sites. We found that the risk of death in colorectal cancer patients was reduced by $12 \%$ when the PNI value was increased 1 unit, however, the $\mathrm{P}$ value was not significant. So, we failed to observe a correlation between PNI and death in colorectal cancer patients (independent protective factors) through the cox model. In addition, for sensitivity analysis, a similar trend was observed using the PNI value as a categorical variable PNI (TERTILE).

\subsection{The analyses for non-linear relationship and the saturation or threshold effects}

Since PNI is a continuous variable, the possibility of a nonlinear relationship between PNI and outcome variables cannot be ruled out. To address nonlinearity of PNI and death, we used the cubic spline function applied to the model, and found that PNI and colorectal cancer patients were non-linear (adjusted histological type, gender, age, platelet count, tumor marker (Figure 3): CA199,CA724,CA242,CEA, TNM staging,

Table 3. The results of multivariate analysis using non-adjusted and adjusted cox regression models.

\begin{tabular}{lccc}
\hline Exposure & $\begin{array}{c}\text { Non-adjusted } \\
\text { model (HR, } \\
95 \% \mathrm{CI})\end{array}$ & $\begin{array}{c}\text { Minimally- } \\
\text { adjusted model } \\
(\mathrm{HR}, 95 \% \mathrm{CI})\end{array}$ & $\begin{array}{c}\text { Full adjusted } \\
\text { model (HR, } \\
95 \% \mathrm{CI})\end{array}$ \\
\hline PNI & $0.94(0.89,1.00)$ & $0.95(0.89,1.01)$ & $0.88(0.78,1.00)$ \\
PNI (TERTILE) & & 1.0 & 1.0 \\
Low & 1.0 & $0.34(0.13,0.85)$ & $0.33(0.08,1.46)$ \\
Middle & $0.33(0.13,0.84)$ & $0.51(0.23,1.13)$ & $0.30(0.07,1.33)$ \\
High & $0.49(0.22,1.07)$ & 0.5139 \\
p for trend & 0.0592 & 0.0764 & 0.1139 \\
\hline
\end{tabular}

Non-adjusted model: None. Minimally-adjusted model: gender; age. Full-adjusted model: histological type, gender, age, platelet count, tumor markers: CA199, CA724, CA242, CEA, TNM staging, postoperative treatment, degree of differentiation, and tumor location.

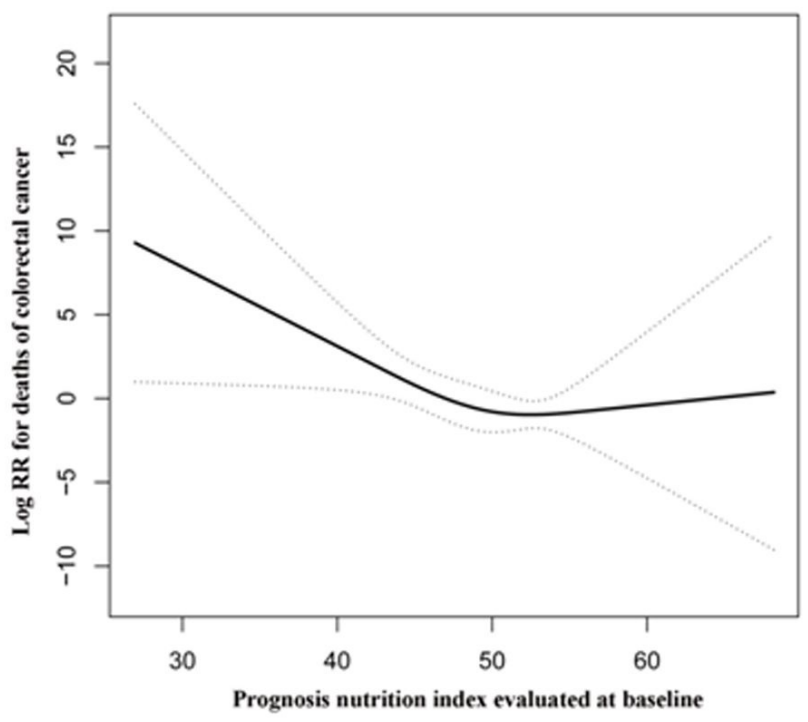

Figure 3. Nonlinear relationship between PNI and survival. postoperative treatment, differentiation, chemotherapy cycle, tumor site). The inflection point was detected by the recursive algorithm, which is 41.35 .Through the two-piecewise cox model, we found that HR of PNI and death had different trends on both sides of the inflection point. On the left side of the inflection point, the effect size, $95 \% \mathrm{CI}$, and $\mathrm{p}$ values were $0.32,0.13-0.79$, and 0.013 , respectively. On the right side of the inflection point, the effect size, $95 \% \mathrm{CI}$, and $\mathrm{p}$ values were 0.84 , $0.62-1.13$, and 0.246 , respectively. The straight line fitting and curve fitting equations were found to vary greatly according to the log-likelihood ratio test. Therefore, the correlation between PNI and death cannot be fitted by straight lines. When the PNI is less than 41.35 , the risk of death is reduced by $68 \%$ for each additional unit of PNI. However, when it exceeds 41.35, even if the PNI increases again, the patient mortality rate cannot be further reduced. The protective effect of PNI on colorectal cancer patients has a saturating effect (Table 4).

\section{Discussion}

Studies showed that PNI is an important prognostic indicator for various malignant tumors (Sun et al., 2014; Chan et al., 2015; Kanda et al., 2011; Fu et al., 2016; Jeon et al., 2016; Migita et al., 2013) Malnutrition is also very common in patients with colorectal cancer, and its incidence is as high as 31.0\% (Gur et al., 2009). The malnutrition is mainly due to preoperative long-term inadequate intake, tumor consumption, tumor complications (such as obstruction, bleeding or perforation), abnormal body metabolism (Jiang \& Zhang, 2016).Therefore, accurate preoperative nutritional status assessment plays an important role in selecting appropriate surgical timing and postoperative adjuvant treatment (Chang et al., 2013).

Preoperative PNI was originally used as a predictor of complications after gastrointestinal surgery and can reflect the nutritional status and immune function of patients (Mohri et al., 2013; Vandenbroucke et al., 2014). The decrease in lymphocyte count during malnutrition suggests abnormal immune mechanisms and decreased anti-tumor immunity (Chang et al., 2013). Plasma albumin concentration can be affected by dietary protein intake, and can be used as an evaluation index of individual nutritional status to a certain extent. Hypoalbuminemia also has a significant correlation with poor prognosis in patients with colorectal cancer. So, PNI value may also be one potential prognosis maker for colorectal cancer.

Table 4. The results of two-piecewise linear regression model.

\begin{tabular}{lccc}
\hline & $\begin{array}{c}\text { Survive } \\
(\mathrm{HR})\end{array}$ & $95 \% \mathrm{CI}$ & p-value \\
\hline $\begin{array}{l}\text { Model I } \\
\text { Linear regression coefficient }\end{array}$ & 0.75 & $(0.57,0.98)$ & 0.033 \\
Model II & & & \\
Inflection point (K) & 41.35 & & \\
$<$ K-segment regression coefficient 1 & 0.32 & $(0.13,0.79)$ & 0.013 \\
> K-segment regression coefficient 2 & 0.84 & $(0.62,1.13)$ & 0.246 \\
Log likelihood ratio test & & & $<0.001$ \\
\hline $\begin{array}{l}\text { We adjusted: histological type, gender, age, platelet count, tumor markers: CA199, CA724, } \\
\text { CA242, CEA, TNM staging, postoperative treatment, degree of differentiation, tumor site. }\end{array}$
\end{tabular}


There is no uniform standard for the boundary value that defines the PNI value in the literature. Some studies (Mohri et al., 2013) used empirical settings of 45 or 46 as the cut-off value, while most studies used the median as the cut-off value, ranging from 45-49.The ROC curve method (Chang et al., 2013) was also used to calculate the PNI value with the best combination of sensitivity and specificity. Yujie Zhao et al (Zhao et al., 2018) empirically set the PNI cutoff value to 48.5 , and found that PNI $>48.5$ was associated with significantly better survival outcomes for independent factors of OS in colorectal cancer patients. Chen Jian-hui (Jian-Hui et al., 2016) used the ROC curve to calculate the PNI cutoff value of 45 . For locally advanced colorectal cancer, the OS of the high PNI group was significantly higher than the low PNI group. In fact, in the correlation analysis, the ROC curve has certain defects in methodology to determine the optimal boundary value. ROC is mainly used to diagnose the type of predictive model, which responds to the descrimination of the diagnostic predictive model. In the correlation analysis, the inflection point of the nonlinear relationship is intended to maximize the difference in effect values between models with different piecewise linear fits (Angerås et al., 2013).

In this study, we found a non-linear relationship between PNI and survival in patients with locally advanced colorectal cancer. and calculated an inflection point of 41.35.On the left side of the inflection point, the risk of death is reduced by $68 \%$ for each unit increase of PNI. On the right side of the inflection point, the patient mortality rate cannot be reduced even if the PNI increases again,

Compared with previous studies, there are some strengthens in the present study. 1) We clarified that PNI had a nonlinear relationship with the prognosis of locally advanced colorectal cancer; 2) We conducted a sensitivity analysis. We converted exposure variables from continuous variables to categorical variables and conducted trend tests to ensure the robustness of the results; 3 ) The regression equation was strictly used to adjust the possible confounding factors; 4) The inflection point was detected using a recursive algorithm, and it was 41.35;5)Our findings was summaried from practical rather than a clinical trial population. Therefore, the results can not be influenced by many artificial factors. Of course, there are some limitations for our study. 1) The samples are geographically restrictive; 2) Only patients with stage II and III were enrolled;3)Some patients were not excluded from the study based on the NAP criteria, but were missing during the follow-up.

In summary, we found that there is not linear relationship between PNI and deaths. In a specific range, PNI can be used to predict the death risk of colorectal cancer.

\section{Acknowledgements}

We would like to thank all colleagues in the Oncology and Anorectal Surgery of the Affiliated Hospital of Guizhou Medical University for their participation in the current research and treatment. This research was funded by a major research project of the Innovation Department of the Ministry of Education of Guizhou Province (QJH-KY-Z [2018] 020).

\section{References}

Akgül, O., Cetinkaya, E., Yalaza, M., Ozden, S., \& Tez, M. (2017). Prognostic efficacy of inflammation-based markers in patients with curative colorectal cancer resection. World Journal of Gastrointestinal Oncology, 9(7), 300-307. http://dx.doi.org/10.4251/wjgo.v9.i7.300. PMid:28808503.

Allegra, C. J., Jessup, J. M., Somerfield, M. R., Hamilton, S. R., Hammond, E. H., Hayes, D. F., McAllister, P. K., Morton, R. F., \& Schilsky, R. L. (2009). American Society of Clinical Oncology provisional clinical opinion: testing for KRAS gene mutations in patients with metastatic colorectal carcinoma to predict response to anti-epidermal growth factor receptor monoclonal antibody therapy. Journal of Clinical Oncology, 27(12), 2091-2096. http://dx.doi.org/10.1200/ JCO.2009.21.9170. PMid:19188670.

Angerås, O., Albertsson, P., Karason, K., Ramunddal, T., Matejka, G., James, S., Lagerqvist, B., Rosengren, A., \& Omerovic, E. (2013). Evidence for obesity paradox in patients with acute coronary syndromes: a report from the Swedish Coronary Angiography and Angioplasty Registry. European Heart Journal, 34(5), 345-353. http:// dx.doi.org/10.1093/eurheartj/ehs217. PMid:22947610.

Chan, A. W., Chan, S. L., Wong, G. L., Wong, V. W., Chong, C. C., Lai, P. B., Chan, H. L., \& To, K. F. (2015). Prognostic Nutritional Index (PNI) predicts tumor recurrence of very early/early stage hepatocellular carcinoma after surgical resection. Annals of Surgical Oncology, 22(13), 4138-4148. http://dx.doi.org/10.1245/s10434-0154516-1. PMid:25801356.

Chang, Y. Z., Cao, J., Tan, W. M., Luo, S. M., \& Zhuang, S. M. (2013). Application of Onodera prognostic nutrition index in prognostic evaluation of elderly patients with colorectal cancer. Zhonghua Wei Chang Wai Ke Za Zhi, 16(6), 561-564. PMid:23801211.

Cheng, Y. L., Sung, S. H., Cheng, H. M., Hsu, P. F., Guo, C. Y., Yu, W. C., \& Chen, C. H. (2017). Prognostic nutritional index and the risk of mortality in patients with acute heart failure. Journal of the American Heart Association, 6(6), e004876. http://dx.doi.org/10.1161/ JAHA.116.004876. PMid:28649089.

Fu, Y., Chen, S. W., Chen, S. Q., Ou-Yang, D., Liu, W. W., Song, M., Yang, A. K., \& Zhang, Q. (2016). A preoperative nutritional index for predicting cancer-specific and overall survival in chinese patients with laryngeal cancer: a retrospective study. Medicine, 95(11), e2962. http://dx.doi.org/10.1097/MD.0000000000002962. PMid:26986105.

Gur, A. S., Atahan, K., Aladag, I., Durak, E., Cokmez, A., Tarcan, E., \& Tavusbay, C. (2009). The efficacy of Nutrition Risk Screening-2002 (NRS-2002) to decide on the nutritional support in general surgery patients. Bratislavské Lekárske Listy, 110(5), 290-292. PMid:19507663.

Heinzl, H., \& Kaider, A. (1997). Gaining more flexibility in Cox proportional hazards regression models with cubic spline functions. Computer Methods and Programs in Biomedicine, 54(3), 201-208. http://dx.doi.org/10.1016/S0169-2607(97)00043-6. PMid:9421665.

Jeon, H. G., Choi, D. K., Sung, H. H., Jeong, B. C., Seo, S. I., Jeon, S. S., Choi, H. Y., \& Lee, H. M. (2016). Preoperative prognostic nutritional index is a significant predictor of survival in renal cell carcinoma patients undergoing nephrectomy. Annals of Surgical Oncology, 23(1), 321-327. http://dx.doi.org/10.1245/s10434-0154614-0. PMid:26045392.

Jiang, Z. Q. H. G., \& Zhang, J. (2016). Effects of serum total lymphocyte number on clinicopathological features and overall survival outcome for cases with rectal cancer who received radical resection. Chinese Journal of Colorectal Diseases, 5, 228-232. 
Jian-Hui, C., Iskandar, E. A., Cai, Sh. I., Chen, C. Q., Wu, H., Xu, J. B., \& He, Y. L. (2016). Significance of Onodera's prognostic nutritional index in patients with colorectal cancer: a large cohort study in a single Chinese institution. Tumour Biology, 37(3), 3277-3283. http:// dx.doi.org/10.1007/s13277-015-4008-8. PMid:26438061.

Kanda, M., Fujii, T., Kodera, Y., Nagai, S., Takeda, S., \& Nakao, A. (2011). Nutritional predictors of postoperative outcome in pancreatic cancer. British Journal of Surgery, 98(2), 268-274. http://dx.doi.org/10.1002/ bjs.7305. PMid:20960457.

Liu, S., Wang, X., Lu, Y., Li, T., Gong, Z., Sheng, T., Hu, B., Peng, Z., \& Sun, $X$. (2013). The effects of intraoperative cryoprecipitate transfusion on acute renal failure following orthotropic liver transplantation. Hepatology International, 7(3), 901-909. http://dx.doi.org/10.1007/ s12072-013-9457-9. PMid:26201928.

Lukaski, H. C., Kyle, U. G., \& Kondrup, J. (2017). Assessment of adult malnutrition and prognosis with bioelectrical impedance analysis: phase angle and impedance ratio. Current Opinion in Clinical Nutrition and Metabolic Care, 20(5), 330-339. http://dx.doi.org/10.1097/ MCO.0000000000000387. PMid:28548972.

Migita, K., Takayama, T., Saeki, K., Matsumoto, S., Wakatsuki, K., Enomoto, K., Tanaka, T., Ito, M., Kurumatani, N., \& Nakajima, Y. (2013). The prognostic nutritional index predicts long-term outcomes of gastric cancer patients independent of tumor stage. Annals of Surgical Oncology, 20(8), 2647-2654. http://dx.doi.org/10.1245/ s10434-013-2926-5. PMid:23463091.

Mohri, Y., Inoue, Y., Tanaka, K., Hiro, J., Uchida, K., \& Kusunoki, M. (2013). Prognostic nutritional index predicts postoperative outcome in colorectal cancer. World Journal of Surgery, 37(11), 2688-2692. http://dx.doi.org/10.1007/s00268-013-2156-9. PMid:23884382.

Nozoe, T., Kohno, M., Iguchi, T., Mori, E., Maeda, T., Matsukuma, A., \& Ezaki, T. (2012). The prognostic nutritional index can be a prognostic indicator in colorectal carcinoma. Surgery Today, 42(6), 532-535. http://dx.doi.org/10.1007/s00595-011-0061-0. PMid:22139087.

Onodera, T., Goseki, N., \& Kosaki, G. (1984). Prognostic nutritional index in gastrointestinal surgery of malnourished cancer patients. Nippon Geka Gakkai Zasshi, 85, 1001-1005.
Peng, J., Zhang, R., Zhao, Y., Wu, X., Chen, G., Wan, D., Lu, Z., \& Pan, Z. (2017). Prognostic value of preoperative prognostic nutritional index and its associations with systemic inflammatory response markers in patients with stage III colon cancer. Chinese Journal of Cancer, 36(1), 96. http://dx.doi.org/10.1186/s40880-017-0260-1. PMid:29268783.

Sun, K., Chen, S., Xu, J., Li, G., \& He, Y. (2014). The prognostic significance of the prognostic nutritional index in cancer: a systematic review and meta-analysis. Journal of Cancer Research and Clinical Oncology, 140(9), 1537-1549. http://dx.doi.org/10.1007/s00432-014-1714-3. PMid:24878931.

Vandenbroucke, J. P., von Elm, E., Altman, D. G., Gøtzsche, P. C., Mulrow, C. D., Pocock, S. J., Poole, C., Schlesselman, J. J., \& Egger, M. (2014). Strengthening the Reporting of Observational Studies in Epidemiology (STROBE): explanation and elaboration. International Journal of Surgery, 12(12), 1500-1524. http://dx.doi.org/10.1016/j. ijsu.2014.07.014. PMid:25046751.

Yang, Y., Gao, P., Chen, X., Song, Y., Shi, J., Zhao, J., Sun, J., Xu, Y., \& Wang, Z. (2016). Prognostic significance of preoperative prognostic nutritional index in colorectal cancer: results from a retrospective cohort study and a meta-analysis. Oncotarget, 7(36), 58543-58552. http://dx.doi.org/10.18632/oncotarget.10148. PMid:27344182.

Yiu, A. J., \& Yiu, C. Y. (2016). Biomarkers in Colorectal Cancer. Anticancer Research, 36(3), 1093-1102. PMid:26977004.

Zhao, Y., Deng, Y., Peng, J., Sui, Q., Lin, J., Qiu, M., \& Pan, Z. (2018). Does the preoperative prognostic nutritional index predict survival in patients with liver metastases from colorectal cancer who underwent curative resection? Journal of Cancer, 9(12), 2167-2174. http://dx.doi. org/10.7150/jca.25346. PMid:29937936.

Zhou, L., Wang, J. Z., Wang, J. T., Wu, Y. J., Chen, H., Wang, W. B., Cao, F., \& Cheng, G. X. (2017). Correlation analysis of MR/CT on colorectal cancer lymph node metastasis characteristics and prognosis. European Review for Medical and Pharmacological Sciences, 21(6), 1219-1225. PMid:28387910.

Zlobec, I., \& Lugli, A. (2008). Prognostic and predictive factors in colorectal cancer. Journal of Clinical Pathology, 61(5), 561-569. PMid:18326017. 